\section{Setting Up a PCR Laboratory}

\section{Carl W. Dieffenbach and Gabriela S. Dveksler}

Division of AIDS, NIAID, HIH, Bethesda, Maryland 20852; Department of Pathology, Uniformed Services University of the Health Sciences, Bethesda, Maryland 20814
PCR technology now permeates all of molecular biology. For many PCR applications, it is essential that the only DNA that enters the reaction is the template added by the investigator. The ideal way to ensure that PCR is performed using only the desired template is to maintain a DNA-free, clean environment. The issue of contamination and the cleanliness required to perform contamination-free PCR has been compared to the good microbiological techniques essential for handling pathogens. ${ }^{(1)}$ The major difference for PCR is that the "biohazard" infects your PCR, not the researcher.

In this section we will describe some general guidelines for the establishment and maintenance of a clean environment for any PCR-based assay system, regardless of the number of samples being processed. As the use of PCR grows in areas such as clinical diagnosis of genetic diseases ${ }^{(2)}$ or the monitoring of viral burden in patients on antiretroviral therapy, ${ }^{(3)}$ rational guidelines for installing a PCR facility will be essential. Our discussion will deal with the establishment of two types of laboratories, one performing contamination-sensitive PCR assays, such as measurement of quantities of target sequences in a sample, and those that are using PCR as a contaminationinsensitive molecular biology tool, such as for mutagenesis of a DNA clone.

The purpose of considering the design, location, and execution of PCR in discrete areas is to plan ahead to avoid contamination of the new PCR reactions with either old PCR products, molecular clones, or sample-to-sample contamination. To date, three approaches have been devised to prevent contamination. The first is the physical separation of the individual parts of the PCR reaction into sample preparation, a pre-PCR location, and a post-PCR location. ${ }^{(4)}$ This approach requires space, money, and supplies to equip and maintain a large infrastructure. However, this is not foolproof because good laboratory practice is still required for the prevention of sample-to-sample contamination. The second method, the use of uracil DNA-glycosylase (UDG) and dUTP substituted for dTTP, is effective only against contamination with dUTP-labeled PCR products. ${ }^{(5)}$ The final method, the use of UV light, is effective against all types of contamination. Its limitation is that this approach cannot destroy all of the contamination, and only reduces it by several logs. Also, it is less effective if the DNA fragment is $<300 \mathrm{bp}^{(6,7)}$ With these caveats in mind, we suggest the following guidelines for establishing a PCR laboratory.

\section{ESTABLISHMENT OF A PCR LABORATORY}

To perform PCR for the repetitive detection of a specific sequence, three distinct laboratory areas are required. The specific technical operations, reagents, and personnel considerations for each one are detailed below. There is new interest in using PCR for the quantitative detection of target sequences, primarily HIV. ${ }^{(3)}$ Thus, as the importance of viral burden measured by RNAPCR grows, the need for contamination-free, reliable PCR becomes essential.

\section{Sample Preparation Area}

This room is specific for sample preparation only. No handling of PCR products or DNA clones containing the sequence to be amplified can take place within this room. Tissue cultures, tissue specimens, and serum samples are all brought into the room and processed for the extraction of DNA or RNA, depending on the application. The tools used in sample processing should not be used for general molecular cloning or manipulation of the target sequence. Aerosols are minimized by briefly centrifuging the tubes prior to opening. Also, the tubes should not be popped open, as this can create an aerosol. The DNA samples should be manipulated with specialized barrier or positive-displacement pipettes, which will prevent the carryover of aerosols 
created during pipetting. Lab coats and gloves should be worn at all times, and gloves should be changed frequently, particularly between each step of the purification process. Additionally, lab coats should be washed frequently.

The method chosen for the purification of the template can have a significant impact on the risk of contamination. In general, the simpler the method that gives reliable results, the better because less sample manipulation will be required. More details about sample preparation will be provided in a future supplement. As one final note, the last step in sample preparation should be vigorous boiling of the DNA samples for up to 15 min prior to moving the samples to the pre-PCR area. The denaturation of template DNA by boiling breaks any large DNA molecules and produces single-stranded DNA, which is a more accessible target for hybridization of the oligonucleotide primers. Excellent results are obtained when the hot start method is used with templates prepared in this manner.

If you or your institution does not have the space for a specific region for sample preparation, consider making arrangements with fellow investigators to share space and the necessary hardware for sample preparation. This arrangement should only be made with colleagues who have never performed molecular cloning with any of the sequences you are interested in amplifying. This ad hoc approach also can work for both the sample preparation and pre-PCR areas.

\section{Sample Preparation and RNA-PCR}

The extra steps associated with RNA-PCR require additional sample handling, providing an increased chance of sample-to-sample contamination. To avoid this problem, the reverse transcription step can be performed in the sample preparation area. The use of UDG with RNA-PCR to prevent contamination has also been reported, ${ }^{(8)}$ and this method is described in Dealing with Contamination: Enzymatic Control of Carryover Contamination in PCR (pp. S10 S14). This approach is particularly valid if the reverse transcription is performed with random hexamer primers rather than the specific antisense oligonucleotide primer. In this method, all of the RNA present in the sample is converted to CDNA with relatively equal efficiency. Because no single specific product is being produced, it is less likely to become a source of contamination. The reverse transcription reaction should be terminated by boiling the reaction, accomplishing both the inactivation of the reverse transcriptase and the denaturation of the RNA:DNA duplex. Alternatively, the RNA strand can be eliminated by RNase $\mathrm{H}$ treatment. ${ }^{(9)}$

\section{Pre-PCR Area}

An area devoted to the preparation of PCR samples is essential. This area must be maintained clean and free of all sources of contamination from molecular cloning and sample preparation. The pre-PCR area must have equipment, specifically both standard micropipettes and positive-displacement types, that are dedicated to the pre-PCR area. The pipettors for setting up the master mixes and the positive-displacement pipettors for adding the samples to each reaction should be carefully stored when not in use. Placement in airtight self-sealing storage bags is an effective way of keeping these free from contamination.

Lab coats that do not leave the pre-PCR area are essential. In addition to equipment, care must be given to the movement of personnel. When experiments are planned or in progress, researchers should not move from the molecular biology (dirty rooms) into the sample preparation and pre-PCR rooms. The same worker, if experienced and careful, can move from the 
sample preparation area to the pre-PCR with the samples and continue the experiment. However, it is probably best if someone else is responsible for the analysis of the results in the post-PCR area.

The pre-PCR area should contain storage space for the reagents needed for PCR, including the positive and negative controls. The aliquots should be quality controlled to be sure that there is no contamination. The negative controls should include all reagents used except input nucleic acid. When positive controls are to be performed, the quantity of nucleic acid being manipulated should be minimized for two reasons. First, to serve as a valid control, a limited number of copies of the target sequence $\left(10^{2}-10^{4}\right)$ must be included in the reaction. Second, by minimizing the amount of DNA, there is less chance of contamination of other samples by DNA aerosol. Because control reactions are essential, the properties of the control template should be considered carefully. The control template should have identical amplification properties with the natural target. In the past, many investigators used a cloned version of the template, which made it very difficult to track contamination of PCR reactions with the positive control. By constructing a control that is both different in size yet maintains the amplification efficiency of the natural target, the problem with contamination with a positive control can be monitored.

The preparation, handling, and quality assurance of reagents will be dealt with in the section on Handling Reagents in the PCR Laboratory (pp. S8-S9). To achieve maximum efficiency and minimize contamination, master mixes containing all of the reagents except the DNA to be amplified and an essential cofactor, such as magnesium or nucleotides, are constructed. If the laboratory is assaying a single product, such as HIV-gag or a limited number of cytokines, master mixes can be aliquoted and sealed under wax. Then, each sample substrate mixed with the correct amount of "the missing key reagent" is pipetted individually into each tube using a positive-displacement pipette. Each tube is opened one at a time and sample is added, again to minimize sample-to-sample contamination. Samples constructed in this way are ready for the PCR machine and, with the use of the wax barrier, both contamination control and a hot start can be achieved. ${ }^{(10)}$

A powerful enzymatic method for elimination of contamination from occurring by PCR product carry over has been devised. ${ }^{(5)}$ All PCR reactions contain the nucleotide deoxyuridine triphosphate (dUTP) at twice the concentration for thymidine triphosphate and the enzyme UDG. This enzyme efficiently removes the uracil moiety from the sugar phosphate backbone by the cleavage of the $\mathrm{N}$-glycosidic bond. This apyrimidinic site then undergoes a beta elimination reaction upon heating. The use of dUTP instead of dTTP in the PCR reaction tags all PCR products as targets for UDG. Standard template DNA or cDNA made using reverse transcriptase is not a substrate for UDG; therefore, this method is nearly $100 \%$ selective. This system is best put in place prior to the appearance of a contamination problem, because it is effective only against nucleic acid that contains uracil.

When using the UDG sterilization method, there are a series of changes in standard operating procedure that must be instituted to achieve optimal performance. First, UDG is included in all master mixes and the standard program on the PCR machine needs to be changed to accommodate this new enzyme. Then, the reactions are incubated at $30^{\circ} \mathrm{C}$ for $5 \mathrm{~min}$ for UDG to remove any contaminating DNA, and then at $95^{\circ} \mathrm{C}$ for $10 \mathrm{~min}$ to inactivate the UDG, followed by the standard PCR cycling. After completion of the PCR, the soak temperature must be set at $63^{\circ} \mathrm{C}$ to keep the UDG inactive. Upon removal from the machine, the residual UDG activity is eliminated by addition of chloroform. This method is foolproof in eliminating PCR product carryover and can greatly streamline the PCR process. Incorporation of this 
method into a PCR application eliminates the need for separate rooms for pre- and post-PCR. However, it must be stressed that this method eliminates only PCR product carryover, not the other forms of contamination.

The third method of contamination control is the use of UV light. This method does not completely eliminate the contamination problem, but rather reduces it several orders of magnitude. However, UV can effectively control contamination arising from molecular clones that are present in the laboratory. Thus, UV serves as a prophylactic treatment of reagents if lowlevel contamination is a problem. The mechanism of how UV light inactivates the template DNA was studied by Sarkar and Sommer. ${ }^{(7)}$ No correlation between the number of thymine-thymine nearest-neighbor events and efficiency of inactivation of the template was seen. However, a correlation was seen with the extent of inactivation and increasing length of the template. To use UV light in the pre-PCR area, master mixes lacking enzyme are exposed to direct UV light in opened tubes. Mineral oil and other reagents can be treated with UV as well. The use of UV in conjunction with the physical separation of pre- and post-PCR areas generally controls both types of DNA contamination.

If you are using PCR only in a contamination-insensitive manner, it is not critical that you maintain a sample preparation area. Often, the substrate in the PCR reaction consists of cloned sequences used in vast excess in the reaction. However, most labs often perform a mixture of contaminationsensitive and -insensitive PCR. Reagents found in all PCR reactions such as nucleotides and Taq DNA polymerase need to aliquoted or purchased in sufficient quantities to insure that there are adequate supplies for the contamination-sensitive reactions. Reagents from the pre-PCR area can always be used for contamination-insensitive reactions; however, once used in the standard molecular cloning lab, these reagents must not return to the pre-PCR area. Reagents for PCR sequencing should also be stored separately from other PCR reaction components.

\section{Location of the PCR Machine}

Where the PCR machine should reside seems like a trivial issue. However, if the thermocycler is to be used for multiple applications, the machine should be located a room where PCR products will be handled. This is a trade-off; you must consider the fact that standard hot start techniques cannot be performed on a PCR machine in a post-PCR area, but the use of Ampliwax (Perkin-Elmer) or its equivalent provides a system for hot start without the opening or manipulation of samples once placed in the machine.

\section{Post-PCR}

After the PCR reactions have been performed, the samples need to be analyzed and the data interpreted. An area should be set aside specifically for post-reaction manipulation of samples. The major source of contamination seen in PCR laboratories is the DNA obtained as product from previous PCR reactions, arising from the microaerosols that are generated during the pipetting and manipulation of the samples. Although these aerosols cause no problems if confined to the post-PCR area, they can cause havoc if they travel to the pre-PCR area on a pair of gloves, in a pipettor, on an investigator, or on a lab coat. If the laboratory has opted not to use the UDG and dUTP system to control PCR contamination, it is extremely critical that there be no mixing of reagents and personnel between this post-PCR and the pre-PCR area. Within the post-PCR area, the tools for analysis should be dedicated to postPCR. If the laboratory is using the 96-well ELISA format for analysis, the specialized equipment (e.g., robotics) could be shared with protein-based or cell culture-based assays but not with anything involved in pre-PCR. If anal- 
ysis is to be performed by gel electrophoresis, then be sure that all the necessary equipment is localized in standard molecular biology areas where contamination-sensitive PCR would not be performed.

From a laboratory management perspective, the division of labor for research projects is critical. Unless a laboratory is involved in the high-volume through-put of hundreds of samples per week, it is not possible to have individuals who work only in the pre-PCR area. The individual responsibilities require that investigators plan to perform the clean work first; then, when that is completed, move on to do the standard molecular biology from which PCR contamination can arise. Consideration also should be given to the establishment of clean days and dirty days. On clean days, sample preparation and pre-PCR experiments will be performed, avoiding molecular biology techniques that are prone to generate contaminating aerosols.

\section{The Perspective of the Small Laboratory}

As a laboratory that is studying the structure, function, and expression of a particular gene, the questions of use of PCR, planning for a pre-PCR area, and staying contamination free require careful thought. If the laboratory is considering establishing a PCR assay for detection of a sequence that has been extensively manipulated within the laboratory, several critical changes must occur. First, there must be a purchase of new equipment, primarily positivedisplacement micropipettors, to dedicate to a pre-PCR area. Sites in rooms where plasmid and phage clones of the sequence of interest have not been manipulated need to be identified. Often a sample preparation/pre-PCR area can be as little as 3-4 linear feet of bench space, a single drawer, and $<1 \mathrm{ft}^{3}$ of freezer $\left(-20^{\circ} \mathrm{C}\right)$ space. Ideally this available space should be on a separate floor from the home laboratory. If such space is not available during normal working hours, the space may be available early in the morning or at night. It is preferable to perform the sample preparation and pre-PCR early before your colleagues unknowingly begin aerosolizing DNA all over the lab.

Anticipation of contamination, acknowledging that it can happen, and acting to prevent it are important first steps to trouble-free PCR. If you have not yet seen contamination in your PCR reactions, there are two possibilities: (1) It is not there or (2) you have not looked hard enough. If PCR is being performed to detect sequences for which molecular clones have been grown and manipulated in the same laboratory or if you are currently performing pre-PCR in the same area as PCR product analysis, try a simple experiment to try to detect low-level contamination. Set up two reactions following your standard procedure, adding template DNA to only one of them, then amplify for 35-40 cycles. If your reaction with no added template is positive, you have a contamination problem. However, sometimes the amount of contamination is so small that it cannot be seen by ethidium bromide staining of the agarose gel and will be detectable only after Southern hybridization and autoradiography.

Dr. John Sninsky has proposed that PCR contamination be considered as a form of infection. ${ }^{(1)}$ If standard sterile techniques that would be applied to tissue culture or microbiological manipulations are applied to PCR, then the risk of contamination will be greatly reduced. Above all else, common sense should prevail.

\section{REFERENCES}

1. Sninsky, J. (pers. comm.)

2. Wang, X. T. Chen, D. Kim, and S. Piomelli. 1992. Prevention of carryover contamination in the detection of beta $\mathrm{S}$ and beta $\mathrm{C}$ genes by polymerase chain reaction. Am. J. Hematol. 40: $146-148$. 
3. Piatak, M. Jr., M.S. Saag, L.C. Yang, S. J. Clark, J.C. Kappes, K.C. Luk, B.H. Hahn, G.M. Shaw, and J.D. Lifson. 1993. High levels of HIV-1 in plasma during all stages of infection determined by competitive PCR. Science 259: 1749-1754.

4. Kwok, S. and R. Higuchi. 1989. Avoiding false positives with PCR. Nature 339: 237-238.

5. Longo, M.C., M.S. Berninger, and J.L. Hartley. 1990. Use of uracil DNA glycosylase to control carryover contamination in polymerase chain reactions. Gene 93: 125-128.

6. Sarkar, G. and S.S. Sommer. 1990. Shedding light on PCR contamination. Nature 343: 27.

7. Sarkar, G. and S.S. Sommer. 1991. Parameters affecting susceptibility of PCR contamination to UV inactivation. BioTechniques 10: 590-594.

8. Pang, J., J. Modlin, and R. Yolken. 1992. Use of modified nucleotides and uracil-DNA glycosylase (UNG) for the control of contamination in the PCR-based amplification of RNA. Mol. Cell. Probes 6: 251-256.

9. 5' Race System Reagent assembly for rapid amplification of cDNA ends. BRL Life Technologies Inc. (catalog number 8374RA).

10. D'Aquila, R.T., L.J. Bechtel, J.A. Videler, J.J. Eron, P. Gorczyca, and J.C. Kaplan. 1991. Maximizing sensitivity and specificity of PCR by pre-amplification heating. Nucleic Acids Res. 19: 3749 . 


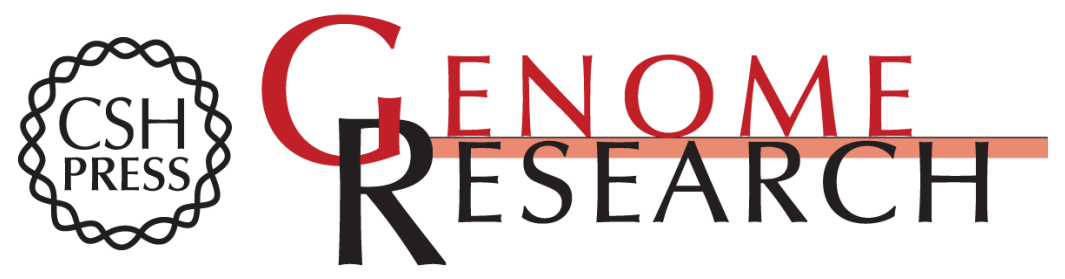

\section{Setting up a PCR laboratory.}

C W Dieffenbach and G S Dveksler

Genome Res. 1993 3: S2-S7

Access the most recent version at doi:10.1101/gr.3.2.S2

References This article cites 8 articles, 1 of which can be accessed free at:

http://genome.cshlp.org/content/3/2/S2.full.html\#ref-list-1

\section{License}

Email Alerting Receive free email alerts when new articles cite this article - sign up in the box at the Service top right corner of the article or click here.

\section{Affordable, Accurate Sequencing.}

To subscribe to Genome Research go to: https://genome.cshlp.org/subscriptions 